\title{
ON THE STRUCTURE OF MAGNETIC FIELD AND ELECTRIC CURRENTS OF A UNIPOLAR SUNSPOT
}

\author{
V. A. KOTOV \\ Crimean Astrophysical Observatory, Crimea, U.S.S.R.
}

\begin{abstract}
This talk deals with the spatial distribution of the total magnetic vector $H$ and of electric currents in the big unipolar sunspot of October 17, 1966.
\end{abstract}

The simple classical model representing the magnetic structure of the sunspot as a 'sheaf' of the diverging field lines, as we have at the top of a solenoid, can not satisfy the recent observations. The appearance of the triplet Zeeman pattern in the umbrae of some spots, the vortex structure and the effect of rotation of the transversal field with height, originally found by Severny (1964), indicate the great complexity of the sunspot magnetic configuration. It is becoming more clear that the magnetic fields of spots have very pronounced fine structure not only along the solar surface but also in the vertical direction.

For detailed study of the field configuration at different geometrical heights in the sunspot we have measured the vector $H$ with the Crimean magnetograph in two spectral lines $\mathrm{Fe}$ I $\lambda 4808$ and $\mathrm{Ca}$ I $\lambda 6103$ simultaneously (Rowland intensities 0 and 9 respectively). The distance between the levels of formation of these lines calculated for the photospherical model is $170 \mathrm{~km}$ (Buslavskij, 1969). The longitudinal field was also measured in the $\mathrm{H} \alpha$ line.

We used the empirical calibration curves found by Severny (1967) which seems to us as most reasonable at the present time.

Figure 1 shows the distribution of the transversal vector $H_{\perp}$ at two levels in the spot. (As the spot has south polarity, the vector directions are drawn for convenience oppositely to real ones; the same is true on Figures 3 and 4). We see essentially azimuthal asymmetry of the field structure at the lower level (where the $\lambda 4808$ line is formed) particularly in the south part of the umbra with $H_{\perp} \approx 4000 \mathrm{G}$. It is also important to note the differences (up to $90^{\circ}$ ) in some parts of the spot between the azimuth of the transversal field determined at two different levels (Figure 2).

The behaviour of the inclination angle $\gamma$ of the total vector at two different levels is illustrated by Figure 3 for two vertical cross-sections $N-S$ and $E-W$ drawn across the spot center. We see a great deal of inhomogeneity of the field directions. An asymmetry is especially pronounced at the lower level $(\lambda 4808)$ for the $S-N$ cross-section. Here the field is mainly horizontal with maximum field strength $\approx 3500 \mathrm{G}$. At the higher level $(\lambda 6103)$ the distribution is more uniform. In the umbra there are some points where the field is almost horizontal (angle $\gamma \approx 50-70^{\circ}$ ); this agrees with some earlier investigations (Nishi, 1962; Iošpa and Obridko, 1965).

The appearance of the azimuthal component of the field at two levels is shown in Figure 4. The clockwise twist of the field lines is stronger at the lower level, pointing 


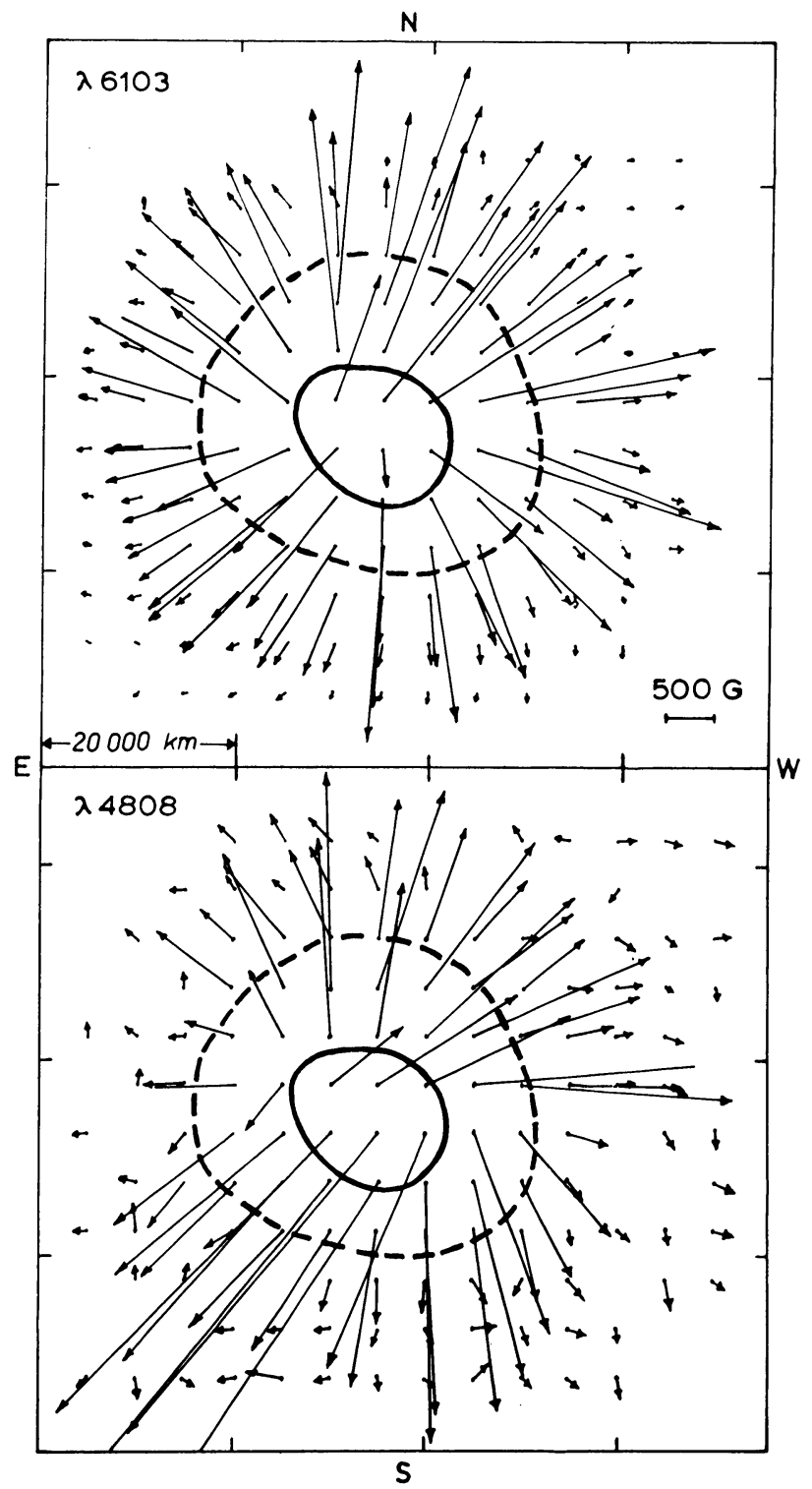

Fig. 1. Transversal field distributions at two levels in the spot.

to the increase of twisting with depth. The same sense of spiraling is observed on the $\mathrm{H} \alpha$-filtergrams taken at the same time.

Our data permit us also to calculate the electric currents in the spot from the relation $\mathbf{j}=c / 4 \pi$ rot $\mathbf{H}$. There is an appreciable difference between the distributions of the vertical currents $j_{z}$ (Figure 5) obtained at two levels. At the lower level there is a large area of electric currents inside the spot flowing upwards, which is surrounded by two regions of downward currents (shaded area). But at the upper level we have only 


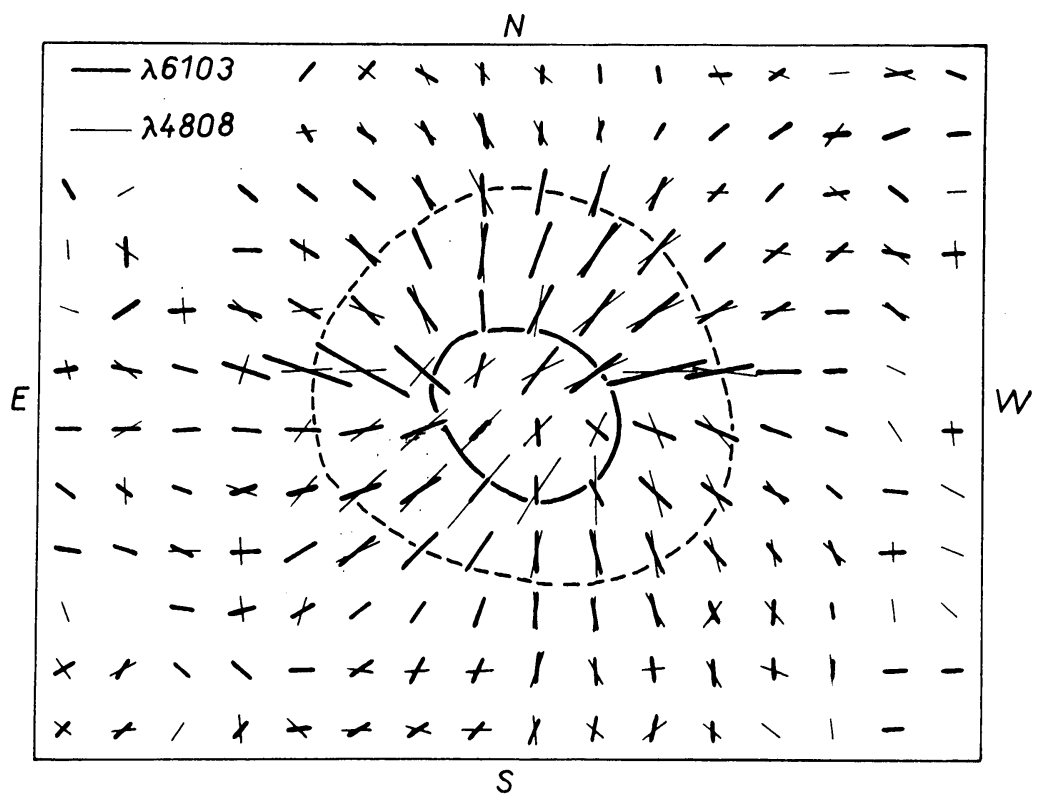

Fig. 2. The comparison of the transversal field directions. 


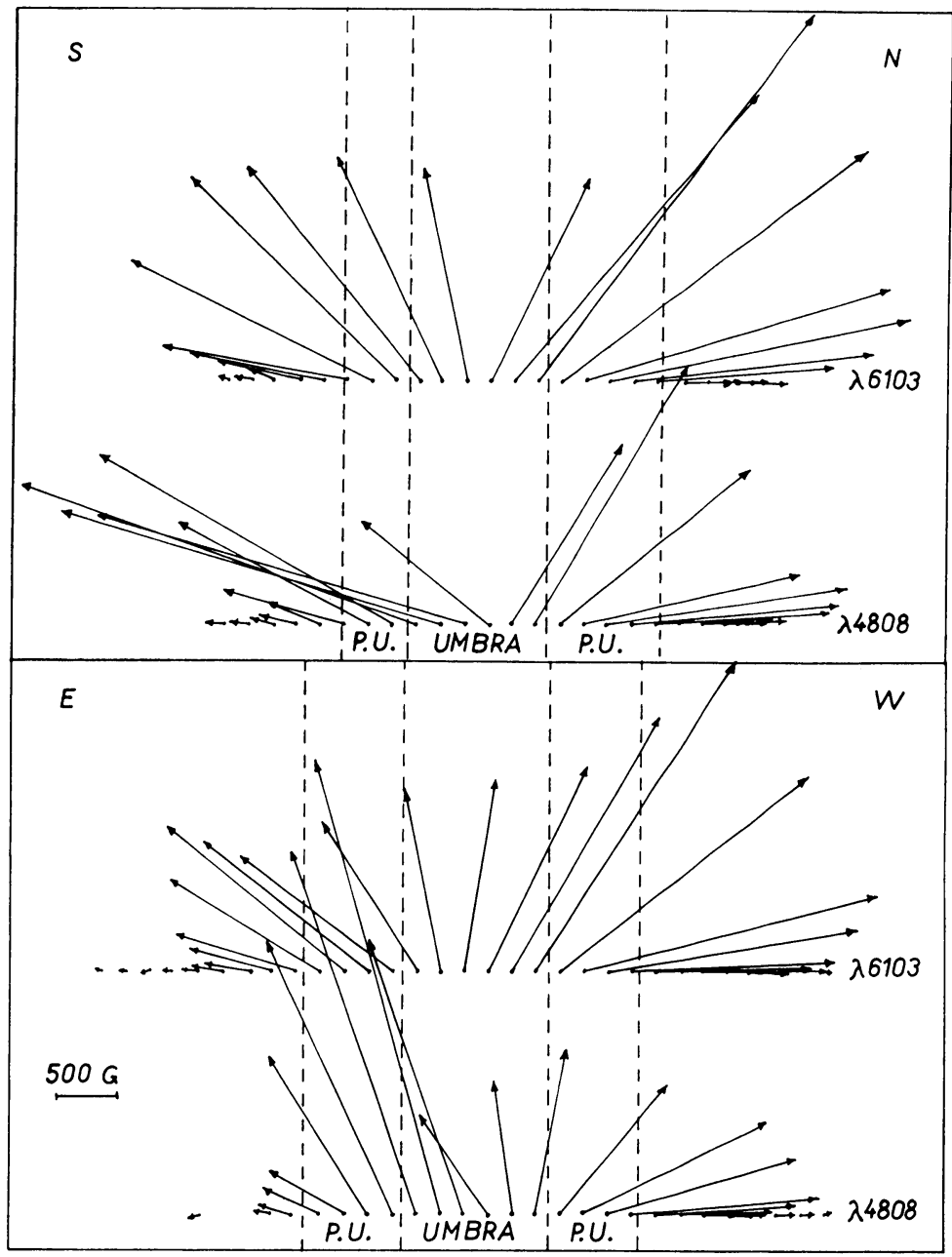

Fig. 3. The field vectors at two levels for two cross-sections drawn across the spot center. 


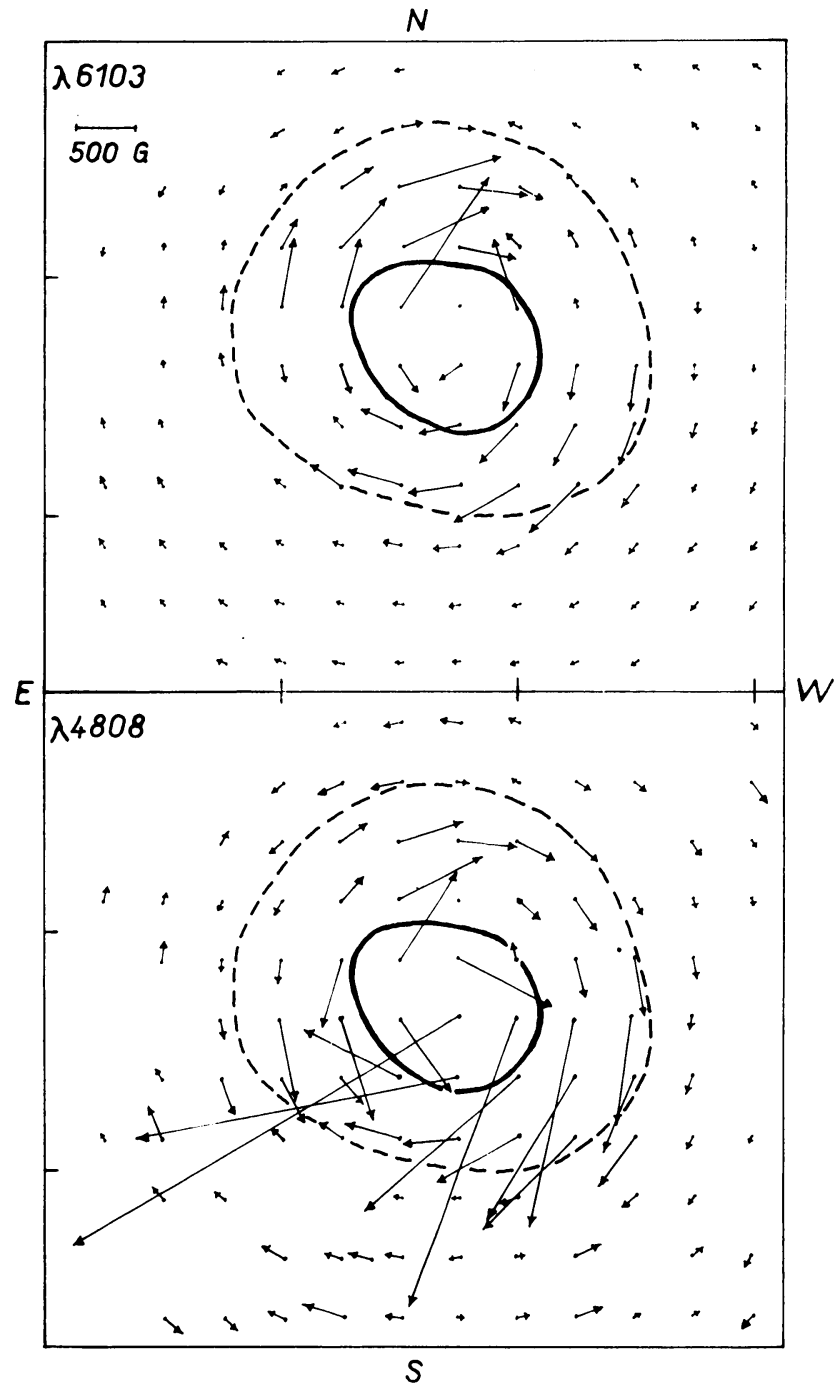

Fig. 4. The azimuthal component of the field. 


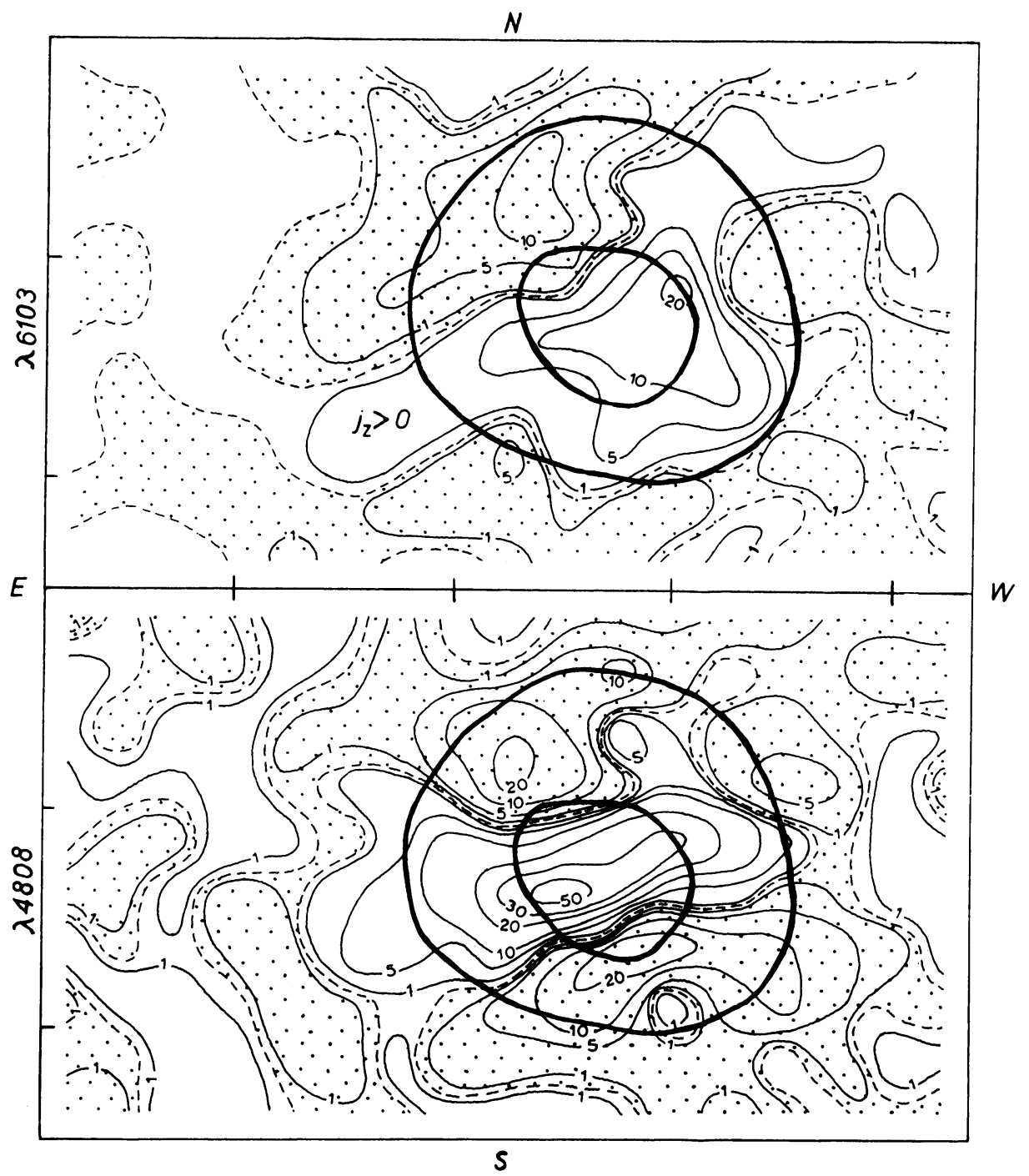

Fig. 5. The maps of the vertical electric currents $j_{z}\left(10^{-2} \mathrm{G} / \mathrm{km}\right)$ inside the spot. 
two areas inside the spot with currents flowing up and down. The value of the vertical current density is $j_{z} \sim 10^{3}-10^{4}$ CGS.

Figure 6 shows the structure of the horizontal electric currents found from measurements of the transversal component $\mathbf{H}_{\perp}$ at two levels. (Note: the calculations show

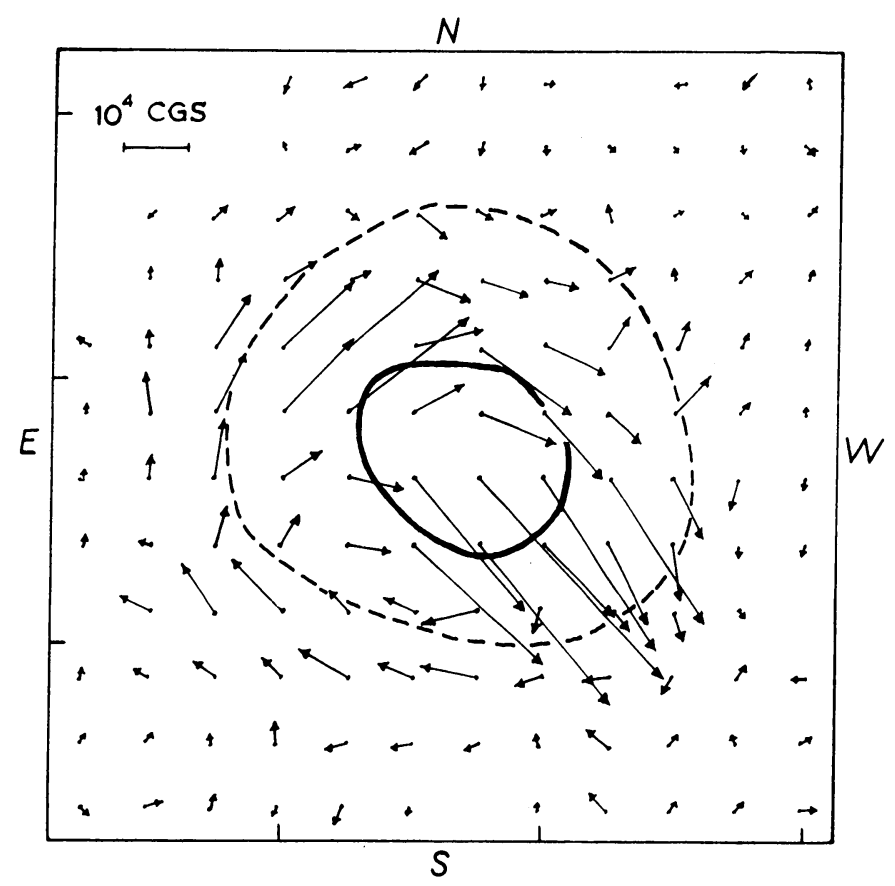

Fig. 6. The structure of the horizontal electric currents.

that the contribution of the derivative $\partial H_{\|} / \partial_{\perp}$ is small compared with the derivative $\left.\partial H_{\perp} / \partial_{\|}\right) .{ }^{*}$ We can see that the electric current system in a spot represents a kind of loop, with the density $j_{\perp} \sim 10^{4} \mathrm{CGS}$ (in the case of a transparent spot model). Regarding the distributions of the vertical and horizontal currents one can imagine their spatial configuration like the current filament emerging in the form of a spiral from deep layers. The total electric current in this filament is about $6 \times 10^{12} \mathrm{~A}$. It is also important to note that the electric currents flow mainly perpendicular to the direction of transversal field; this is not in favor of the force-free character of the magnetic field. Using the photographic method Rayrole and Semel (1970) have come recently to the same conclusion on the field structure of a sunspot.

A calculation of magnetic force shows that it is $\sim 10^{-3} \mathrm{~d} / \mathrm{cm}^{3}$ and it is directed mainly along the solar longitude and downwards inside the spot. This is accompanied by an inflow of gases as observed in the $\lambda 6103$ line.

* See footnote on following page. 


\section{References}

Buslavskij, V. G.: 1969, Izv. Krymsk. Astrofiz. Obs. 39, 317.

Iošpa; B. A. and Obridko, V. N.: 1965, Soln. Dann. No. 3, 54.

Nishi, K.: 1962, Publ. Astron. Soc. Japan 14, 325.

Rayrole, J. and Semel, M.: 1970, Astron. Astrophys. 6, 288.

Severny, A. B.: 1964, Izv. Krymsk. Astrofiz. Obs. 31, 126.

Severny, A. B.: 1967, Izv. Krymsk. Astrofiz. Obs. 36, 22.

\section{Discussion}

Schröter: (1) The two lines used by Kotov are supposed to originate in different heights, therefore they will show a different sensitivity to temperature. As we learned here calibration errors for magnetographs are a direct function of the line's temperature sensitivity. How did Kotov assure that such errors do not occur?

(2) Have you now at your observatory enough material to decide whether the observed rotation of the field azimuthly with depth does depend on the hemisphere or on the polarity of the spot?

Severny: (1) I think it is too premature to reconsider the problem of calibration due to the effects of temperature sensitivity without thinking it over and without checking it by further observations.

(2) We have a lot of material for the unipolar spot under consideration covering all of the passage from the east to the west limb and for two rotations. This material is now being reduced and considered by Kotov.

Brueckner: What is the optical depth of the two lines used for these measurements?

Severny: I do not remember exactly, but I think that for the deep seated line $\lambda 4808, \tau$ is about 1.2 and for the line $\lambda 6103$ it is about 0.3 .

Semel: I think you are really very lucky to obtain the magnetic field measurements in three dimensions. Could you test if your measurements yield $\nabla \cdot H=0$ ?

Severny: Kotov has made this check with div $H=0$ and there is as usual in these cases disagreement between the $\partial H / \partial z$ found from this equation and that found by direct measurements.*

* This fact points at the difficulties of the usual interpretation of the $H$-recordings in spots. We have discussed recently (Kotov, V. A.: Publ. Crim. Astron. Obs. 46, in press) the considerable discrepancy between the height gradients of the vertical field $\partial H_{z} / \partial z$ obtained in two ways: (a) from the measured vertical field at two levels $(\sim \pm 3 \mathrm{G} / \mathrm{km})$ and (b) from the use of the equation $\operatorname{div} H=0(\sim \mp 0.5$ $\mathrm{G} / \mathrm{km}$ ). This discrepancy leading to seeming violation of the equation div $H=0$ could be mostly attributed to the great difference between very high depth- and low surface-resolutions. Here one should take into account the fine structure of the field which is less than the resolving power of the instrument. 